\title{
Riscrivere i Vangeli tra eclissi e ritorno del sacro: sei riscritture italiane dei Vangeli
}

Elisabetta Lo Vecchio

\section{(2) OpenEdition}

\section{Journals}

Edizione digitale

URL: http://journals.openedition.org/cei/219

DOI: $10.4000 /$ cei. 219

ISSN: 2260-779X

Editore

UGA Éditions/Université Grenoble Alpes

\section{Edizione cartacea}

Data di pubblicazione: 15 juillet 2009

Paginazione: 267-276

ISBN: 978-2-84310-145-8

ISSN: $1770-9571$

Notizia bibliografica digitale

Elisabetta Lo Vecchio, «Riscrivere i Vangeli tra eclissi e ritorno del sacro: sei riscritture italiane dei Vangeli», Cahiers d'études italiennes [Online], 9 | 2009, online dal 15 janvier 2011, consultato il 27 mars 2021. URL: http://journals.openedition.org/cei/219 ; DOI: https://doi.org/10.4000/cei.219 


\title{
RISCRIVERE I VANGELI TRA ECLISSI E RITORNO DEL SACRO: SEI RISCRITTURE ITALIANE DEI VANGELI
}

\author{
Elisabetta Lo Vecchio
}

Università di Bologna

Per comprendere a pieno il ruolo che la religione ha nella cultura italiana della fine del Novecento, e, più in specifico, nella narrativa, proporrei una definizione del "senso del religioso" partendo dal concetto di religiöse Sinn dell'idealismo tedesco, come dimensione essenziale della natura umana, anche là dove, vedi l'ateismo della modernità, tale dimensione è negata. L'uomo è homo religiosus, così com'è stato messo in evidenza da Rudolf Otto. Anche nell'epoca della presunta emancipazione dal sacro, l'uomo si pone sempre a confronto con la religione in positivo come in negativo. Non è, quindi, un caso che proprio negli anni in cui la cultura italiana si crede libera dal giogo della vetusta metafisica e della religione, di fronte al rinnovarsi della Chiesa post-conciliare, in campo letterario persista la presenza della religione come tema. La figura e l'opera di Mario Pomilio sono sicuramente, da questo punto di vista, esemplari. Così negli Scritti cristiani (1979) egli oppone al "dogmatismo del rifuto" dei "settori non cristiani" il "liberatorio discorso sul Cristo", che tiene, invece,

accese le esigenze metafisiche e gli interrogativi intorno al senso dell'esistenza [...], lascia aperto il versante della problematicità, e con ciò stesso introduce in una storia tutta dominata dai vari scientismi e dai vari materialismi un reattivo che porta a salvamento ciò che è irrinunciabile, l'uomo stesso, l'uomo come senso, come valore, come fine ${ }^{\mathrm{I}}$.

"Le esigenze metafisiche" sono per Pomilio parte stessa dell'essenza dell'uomo, la cui rinuncia porta a conseguenze ben poco gaie, per non dire tragiche. La storia degli ultimi venti anni sembra avergli dato ragione: il ritorno del sacro in forme violente e prevaricatrici è proprio il segno

I. M. Pomilio, Scritti cristiani, Milano, Rusconi, I976, p. 60. 
di questo legame imprescindibile che vincola l'uomo al bisogno di trascendenza.

Cosa significa dunque riscrivere il Vangelo tra il trionfo della secolarizzazione e il ritorno del sacro? Ricercare un senso perduto, un'identità perduta? La ricerca di senso attraverso forme e strutture dell'immaginario preesistenti, come può essere il Vangelo o il mito, è una strada già percorsa nella storia della letteratura. Il Cristo secolarizzato si presta fortemente a un'interpretazione in prospettiva antropologica. Per Cristo secolarizzato intendiamo il Cristo frutto di una progressiva de-sacralizzazione, il Cristo non più vere homo et vere Deus, ma l'archetipo dell'uomo inabitato da Dio e il Cristo pasoliniano tantum homo maestro di saggezza. La ricerca di questa presenza di Dio nella coscienza come ricerca del vincolo originario tra l'uomo e Dio è, appunto, l'elemento che fa di Gesù l'immagine paradigmatica dell'uomo alla ricerca di senso. Il Cristo secolarizzato è Figlio di Dio, Logos incarnato, come tutti gli uomini. In questo senso la sua storia è per l'età moderna e contemporanea emblematica: Cristo è l'immagine mediatrice e risolutrice del rapporto interrotto tra l'uomo e Dio non solo sul piano dell'esistenza, ma anche su quello dell'immaginario. La sua vicenda insegna, esemplifica, più profondamente del mito, la storia dell'uomo, nelle sue cadute e nei suoi avanzamenti nei confronti di Dio, ovvero "l'uomo come senso" (ibidem, p. 62), senso e fine della creazione. Riscrivere il Vangelo significa, dunque, riscrivere la storia dell'uomo, come ricerca di senso, senso che si realizza, appunto, direbbe Schleiermacher, nella coscienza inabitata da Dio e nell'insegnamento morale di colui che prima di ogni altro, più di ogni altro, ha rappresentato l'uomo realizzato nella sua finitezza.

Alla luce di queste premesse i sei testi presi in esame - L'opera del tradimento (1975) di Mario Brelich, Il Quinto Evangelio (1975) di Pomilio, La Gloria (1978) di Giuseppe Berto, Getsemani (1980) di Giorgio Saviane, La notte del lupo (1989) di Sebastiano Vassalli e Il Vangelo di Giuda (1989) di Roberto Pazzi - sono sicuramente testimonianze di questa crescente esigenza di senso in un'epoca priva di fondamento. In maniera diversa i sei autori elaborano attorno alla vicenda di Gesù una struttura narrativa che mira a ricostruire quello che Pomilio definisce come "il quinto evangelio", fino alla denuncia dell'impossibilità di tale ricerca da parte di Pazzi. Il "quinto evangelio" è il Vangelo mai rinvenuto, e, forse, mai scritto, ma borgesianamente ricercato, è il Vangelo che ciascuno vorrebbe scrivere, il Vangelo di ciascuno. Il potenziale epico dei Vangeli è, come hanno osservato sia Borges che Pomilio, infinito. "Ciascuna generazione," rileva Pomilio, "ha potuto avvicinarli come se si trattasse di libri appena 
apparsi riaprendo da capo il discorso intorno ad essi e quasi, in pratica derivandone un suo vangelo" (ibidem, p. III). Da qui ha origine l'idea del "quinto evangelio [...] il libro nascosto il quale soggiace in perpetuo ne modifica e ne amplifica il senso, trasformandone la verità in una sorta di meta mobile" (ibid.), idea, tutta moderna e ben poco cattolica, di un'ermeneutica individuale della Parola. Secondo una concezione secolarizzata della Scrittura, esso è il Vangelo di ciascuno, vale a dire il Vangelo di cui ogni individuo si appropria, dandone una versione che va a colmare, solo in parte, gli interstizi vuoti nelle pieghe del racconto canonico. Le sei riscritture prese in esame sono anch'esse parte di questo Libro, tasselli che vanno a completare il quadro inattingibile del "quinto evangelio", "il libro dei Libri o l'Apocrifo degli Apocrifi [...] perpetuamente inseguito e perpetuamente nascosto" (ibid.). A un primo sguardo ciò che più colpisce è il rilievo che tutte e sei danno a una figura che la cristianità da sempre ha tenuto ai margini della vicenda di Gesù, quella del traditore, Giuda Iscariota. L'immaginario moderno da Thomas De Quincey (XIX sec.) in poi ha riscoperto il vincolo originario tra la vicenda di Gesù e quella del suo traditore: senza Giuda non possono esservi, infatti, né Passione né resurrezione. Il male ha dunque avuto un ruolo decisivo all'interno dell'opera di redenzione: Dio ha abbandonato il Figlio sulla croce, come Giuda ha tradito il suo maestro, tutto in vista della salvezza del genere umano. Fu allora vero tradimento?

L'opera del tradimento di Brelich sembra rispondere a tale interrogativo. Il romanzo è il resoconto di una meticolosa indagine poliziesca, compiuta da un celebre personaggio della letteratura noir, l'investigatore Auguste Dupin di Edgar Allan Poe, sul come e il perché del tradimento in rapporto all'opera di redenzione. Costruito su due piani temporali, l'illud tempus dei Vangeli e l'bic et nunc di Dupin, L'opera è una trasposizione secondaria in cui i Vangeli, l'ipotesto, rimangono sullo sfondo per diventare oggetto di interpretazione, mentre la diegesi si struttura come una glossa. A conclusione del romanzo l'autore inserisce una riscrittura del racconto evangelico dell'ultima cena in forma di novella, redatta dallo stesso Dupin, come a tirare le somme dell'inchiesta attraverso la messa in scena in presa diretta dell'opera del tradimento. Nello sviluppo della narrazione l'inchiesta sul tradimento si amplia fino a diventare un'inchiesta su Dio, fino all'elaborazione di una teologia che si rivela come una metafisica del male: il male, a rigor di logica secondo Dupin, è l'imprescindibile condicio sine qua non del bene, il tradimento della redenzione. Ma il discorso di Brelich non è, al contrario di quello di Berto e Pomilio, un discorso sul rapporto tra l'uomo e Dio, bensì un discorso sull'uomo, sul suo immaginario e sui suoi 
tabù, con forti echi freudiani e junghiani. Dio e Gesù sono qui rappresentati come proiezioni dell'inconscio collettivo, come archetipi dell'immaginario. Il conflitto in atto tra il Padre e il Figlio è la rappresentazione sul piano metafisico del dramma umano troppo umano del complesso edipico, portato a estreme conseguenze. Brelich utilizza il racconto evangelico considerandolo un mito nel senso freudiano del termine. Il sacro diventa, quindi, secondo i parametri dell'antropologia contemporanea, oggetto di studio per comprendere l'uomo e il suo rapporto con l'altro da sé, con quell'entità che l'uomo, autoalienandosi, chiama Dio, ma che, secondo quanto affermava Feuerbach, non è altro che una proiezione di se stesso. Il dramma di Giuda è appunto il dramma dell'uomo tradito da questa entità esterna che è l'uomo stesso.

L'attività ermeneutica che ruota attorno ai testi biblici, "infinita", sembra perciò diventare centrale. $R i$-scrivere il Vangelo significa prima di tutto $r$-leggerlo e $r i$-ascoltarlo, coglierne echi nascosti, $r$-interpretarlo attraverso la rappresentazione. In un'epoca ermeneutica come quella postmoderna, ri-scrivere il Vangelo non s'identifica più soltanto nella messa in scena della vicenda di Gesù, lasciandosi dietro le spalle il lavoro interpretativo da cui trae origine la rappresentazione, ma svelare in primis quell'attività ermeneutica, "infinita", che produce la rappresentazione, mostrarla. È così che, come L'opera del tradimento, Il Quinto Evangelio di Pomilio è prima di tutto il racconto di una quête dell'autentico significato del messaggio di Gesù, una ricerca non solo filologica e storica ma anche esistenziale, intrapresa dall'americano Peter Bergin nelle maglie del tempo e dei testi, nell'utopico progetto di riportare alla luce il Quinto Vangelo. Dietro le quinte del romanzo troviamo il Concilio Vaticano II, come lo stesso Pomilio ha ammesso. La centralità della Scrittura riaffermata dal Concilio diventa il motore della narrazione: solo nei quattro Vangeli storici possiamo ricostruire in sintesi l'autentico messaggio di Cristo, "il quinto evangelio". L'avventura ermeneutica di Bergin ha dunque inizio e compimento nei Vangeli. Il "quinto evangelio" non è che l'Urtext che soggiace ai quattro testi, l'insegnamento, le parole di Cristo, il Cristo stesso che è il Logos incarnato. Il dramma teatrale Il Quinto Evangelista, che va a concludere il romanzo, è appunto questa Parola che si fa carne, si fa rappresentazione. Si tratta di una transattualizzazione dei Vangeli: esso ha per protagonisti personaggi della contemporaneità, i quali, in una sala parrocchiale nel I940, mettono in scena la Passione, assumendo ciascuno le vesti di un per-

2. P. C. Bori, L'interpretazione infinita, Bologna, Il Mulino, 1987. 
sonaggio del Vangelo. Il dramma si configura pirandellianamente come una doppia messa in scena, come un dramma nel dramma, in un riflusso continuo tra l'bic et nunc della diegesi e l'illud tempus della Passione. Lo spirito pirandelliano che lo anima si palesa nel finale, con il disvelamento dell'identità dell'enigmatico personaggio del quinto evangelista, il quale non è che Cristo. Principale interlocutore di Gesù è Giuda, un Giuda rabbioso e polemico nei confronti della sua predestinazione al male. Pomilio mostra il lato umano di Giuda, dimensione che ritroveremo in seguito in Berto. Giuda rivendica con rabbia la salvezza promessa dal Cristo, e si rivolge spontaneamente all'evangelista-Gesù. Giuda è condannato non per aver tradito, ma per la sua aridità di cuore, spiega l'evangelista, agostinianamente per la mancanza di fede nella misericordia divina, in nome della diffidenza e dell'orgoglio. Proprio in Giuda si attualizza e s'incarna la condizione della società moderna nei confronti di Dio, affamata d'amore, ma incapace di corrisponderlo a sua volta. Il Cristo redivivus di Pomilio, celato dietro la maschera del quinto evangelista, ha, quindi, il compito di riportare alla luce il senso originario del suo messaggio, per riappropriarsi del ruolo di mediatore tra Dio e l'uomo, in una società, come quella contemporanea, che ha ghettizzato Dio, condizione che Pomilio negli Scritti cristiani definisce come "apartheid di Dio". La ricerca storico-filologica del Quinto Evangelio non è altro che la riscoperta del kerygma così come Gesù lo ha dettato. Il senso perduto si manifesta finalmente in quel dialogo concitato tra Giuda, portavoce dell'umanità contemporanea, e Gesù, l'unico, in realtà, a poter scrivere il Quinto Vangelo, in quanto Verbo divenuto carne.

Porta al paradosso l'identificazione della Parola divina in Gesù Pazzi nel Vangelo di Giuda, romanzo in cui il mito del Quinto Vangelo si disvela come un'utopia. Parafrasando Nietzsche, per Pazzi non c'è un Urtext, ma solo interpretazioni. Ognuno offre una propria versione della Parola divina, del Cristo, in totale dissenso con le altre. È così che Pazzi elabora un romanzo polifonico a struttura polisemica, in cui la vicenda di Gesù viene narrata da più voci all'imperatore Tiberio in un collage di tre racconti che hanno esiti finali differenti. Quale versione è più attendibile? Non c'è risposta. Dietro al moltiplicarsi dei punti di vista e delle voci si cela il reale intento dell'autore, mettere in scena la scrittura nella sua essenza mortifera. La parola scritta è, infatti, un oggetto plasmabile. Ogni narratore modifica anche involontariamente la realtà degli eventi raccontati. La scrittura è, quindi, secondo Pazzi, tradimento, un tradimento più grave di quello di Giuda: l'ironia dell'autore si scaglia evidentemente contro quel potere demagogico che fa della parola scritta una minaccia alla libertà. Il 
Vangelo di Giuda è, rispetto agli altri testi presi in esame, un romanzo postmoderno, in cui l'autore gioca con l'archetipo letterario di Cristo, con il Cristo quale Verbo incarnato, per decostruire la storia narrata nei Vangeli, moltiplicando le voci e i finali, al fine di dimostrare il proprio assioma, la scrittura come morte. E in effetti la scrittura dei Vangeli viene dopo la morte di Cristo (d.C.). Se la Parola divina s'incarna e muore in croce non può che risorgere moltiplicandosi e contaminandosi. Pazzi risponde così all'enigma degli enigmi, perché Gesù non abbia mai scritto un suo Vangelo. "Il grande tradimento verso la vita," scrive Pazzi, "è scrivere"3, in quanto la scrittura è sempre menzogna e falsificazione.

Il discorso sul rapporto tra l'uomo contemporaneo e Dio è centrale nella Gloria di Berto. La scelta del punto di vista, quello del traditore, si accorda perfettamente con l'idea derivata dal libro dell'Apocalisse della realtà della morte come autentico messaggio di Cristo. La genesi della Gloria è preceduta da un periodo di circa dieci anni, durante il quale Berto si approssima in due occasioni alla figura di Cristo: in L'uomo e la sua morte (1962), un testo teatrale dalla scrittura poco fluida e piuttosto retorica, e nell'atto unico con prologo La Passione secondo noi stessi (1972). La scelta del teatro come luogo per rappresentare la figura di Cristo è sicuramente indicativa, non solo per il richiamo alla tradizione dei Misteri medievali, ma, soprattutto, per l'immediatezza offerta dalla rappresentazione scenica così affine al realismo dei Vangeli. Nel personaggio centrale di L'uomo e la sua morte, il bandito Salvatore, tradito dal suo luogotenente, Berto proietta l'immagine di Gesù tradito da Giuda. Lo stesso Salvatore, poco prima di essere consegnato ai carabinieri, s'interroga sulle ragioni per cui Giuda tradì Cristo. Il dilemma della ragione autentica del tradimento ritorna in La Passione secondo noi stessi non più dal punto di vista di Cristo-Salvatore, ma da quello di Giuda. Il dramma di Giuda nasce da una profonda lacerazione tra il sentimento di amore nei confronti di Gesù e il proprio agire. La disperazione e l'angoscia del traditore animano l'ultima parte del dramma, in un crescendo di pathos fino al grido lacerante del traditore all'udire le ultime parole di Gesù sulla croce. Costruita, come Il Quinto Evangelio, su due piani temporali differenti e lontani, l'illud tempus del Vangelo e l'hic et nunc dell'oggi, La Passione mette in scena il confronto tra i personaggi della contemporaneità, un sociologo, un giurista, uno psicoanalista, e l'autore, e il racconto dei Vangeli mediante i loro personaggi, il confronto tra la società contemporanea e il messaggio

3. R. Pazzi, Il Vangelo di Giuda [1988], Milano, Baldini e Castoldi, I999, p. 226. 
di Cristo. Anche qui come in Pomilio la figura di Giuda non ha un valore meramente storico-letterario, bensì simbolico. Nel suo dramma viene, infatti, proiettata, come in Pomilio, l'immagine dell'umanità contemporanea, tragicamente condannata a non comprendere a pieno i disegni divini.

Nella Gloria è Giuda stesso a narrare in prima persona la propria Bildung di giovane rivoluzionario ebreo, figlio di un mercante di Gerusalemme, posto di fronte alla scoperta del valore supremo della morte, che è poi l'unico punto di vista che per Berto dia senso alla vita e stabilisca un rapporto religioso tra la vita e la morte. La tenacia con cui Gesù persegue il suo disegno di morte colpisce Giuda, come già Berto, il quale in $\mathrm{La}$ mia lettura dei vangeli (1966) scriveva: "La prima cosa che salta fuori evidente dalla lettura dei vangeli è la volontarietà della morte di Cristo: egli è andato alla propria morte si potrebbe perfino dire con spavalderia, con ostinazione, e almeno fino a un certo punto, con straordinaria fermezza ${ }^{4}$." Attorno al tema della morte si sviluppa quindi il Vangelo secondo Giuda di Berto. Il messaggio apostolico di Giuda non è la vita eterna, bensì la morte come unica realtà della condizione umana. Già Pasolini, nel Vangelo secondo Matteo (1964), poneva l'accento sulla morte come realtà unica dell'essere umano. Berto conclude il suo racconto con l'immagine della crocifissione del Figlio di Dio, l'uomo, abbandonato da Dio al suo autentico destino, in quanto essere finito, in quanto creatura. Il crocifisso non è l'immagine di Dio che muore e risorge, ma la tragica scoperta di Dio come morte. L'autentica opera di redenzione è, allora, il disvelarsi della morte come unico destino dell'uomo. È questa la Gloria promessa dalle Scritture: la morte, l'oblio, il ritorno all'Uno. Giuda, e come Giuda ogni uomo, pur avendo fede in Dio, non può e non sa capire. Le parole del sedicente Dio incarnato sono misteriose ed enigmatiche, i suoi atti spesso contraddittori, e l'ombra dell'inganno si annida ovunque. Tuttavia, l'ultimo atto d'amore di Giuda è la rinuncia a se stesso e alla logica in nome della fede. Per sempre il suo nome sarà macchiato dall'infamia di un tradimento che non ha deciso di compiere, poiché parte di un oscuro progetto del suo Dio. A un livello ulteriore è possibile rileggere La Gloria non solo come una rappresentazione della tragedia dell'esistenza contemporanea, ma soprattutto come un rovesciamento parodico di tutto ciò che la tradizione cristiana dai Vangeli in poi ha affermato sulla vicenda di Gesù. Non è forse possibile intendere Giuda non più come il traditore per eccellenza,

4. G. Berto, "La mia lettura dei vangeli", Il Resto del Carlino, I8 dicembre 1966. 
ma come un uomo tradito, tradito da Dio e dal suo Figlio? È dunque l'impossibilità di un confronto tra i disegni imperscrutabili di Dio e il senso di una singola e finita esistenza umana che Berto mette in scena, attraverso il punto di vista del reietto. La tragica condizione dell'uomo è rappresentata dall'incapacità di comprendere ciò che l'Eterno ha in serbo per lui, e, in primis, ciò che l'Eterno è. L'unica soluzione sembra essere paradossalmente la fede in un Dio che è pronto in ogni momento a tradire il suo unico Figlio, l'uomo.

L'assenza della Chiesa all'interno dei testi appena presi in esame non è certamente casuale. Tale silenzio ha il valore di un'aspra critica rivolta a un'istituzione ritenuta ormai obsoleta e fuori dallo spirito di un'epoca emancipata dai dogmi. La necessità di una fede senza Chiesa, di un Vangelo Eterno così come già affermava Lessing, è per la cultura post-moderna fondamentale. Così Giorgio Saviane in Getsèmani e Sebastiano Vassalli in La notte del lupo sembrano proporre un modello di religiosità e di fede, un "senso religioso" svincolato dalle istituzioni storiche, puramente spirituale e morale, una religione senza Chiesa. Cosa rimane del cristianesimo se non il messaggio d'amore, racchiuso nel Discorso della montagna, tanto caro a Bonhoeffer? "L'etica," rileva Carmelo Dotolo, per la cultura post-moderna "[...] è rottura e distacco dall'attaccamento a se stesso, nell'esercizio arduo, quasi ai limiti dell'impossibile, dell' amore dell'altro e per l'altro s". Il tema centrale di Getsèmani è appunto l'amore per l'altro, la charitas e la pietas perseguite a costo del sacrificio. È, quindi, nella figura del Cristo del Calvario che Saviane rinviene il modello di comportamento a cui conformarsi: "il dolore [...]," afferma il Cristo di Saviane, "è forse l'unica emozione di Dio per essere, per sentire di esistere. Senza il dolore non sapresti della felicità ${ }^{\prime \prime}$. Al bene assoluto come amore e charitas, incarnato in un Gesù moderno, Saviane oppone il male come invidia e rancore, rappresentato paradigmaticamente dall'immagine dello storpio guarito, sul quale l'autore proietta la figura di Giuda. La scelta di Saviane di modernizzare la vicenda di Gesù, collocandola in un orizzonte temporale contemporaneo al lettore, mira ad attualizzare la figura del Figlio di Dio e il suo messaggio inteso esclusivamente in orizzonte etico. Il "cristianesimo possibile" di Saviane è, infatti, un cristianesimo senza Chiesa, e, quindi, senza sacro. Analogamente Vassalli, mediante una narrazione a focalizzazione interna nella coscienza di Giuda, oppone il messaggio originario di

5. C. Dotolo, Un cristianesimo possibile. Tra post-modernità e ricerca religiosa, Brescia, Queriniana, 2007, p. II9.

6. G. Saviane, Getsèmani, Milano, Mondadori, I980, p. I45. 
Gesù, rappresentato dal traditore, al messaggio del cristianesimo storico della Chiesa, erede della tradizione ebraica. Come Caino in Genesi, così Giuda è condannato a errare nel tempo imprigionato nel proprio ruolo di vendicatore, "immemore di ciò che era stato nel passato 7 ". L'ultima maschera da lui assunta è quella dell'attentatore di Giovanni Paolo II, il lupo grigio Alì Agca. La notte del lupo è l'eterna notte in cui il traditore si converte e ripudia il ruolo del traditore assegnatogli dai Farisei, per vendicarsi dell'autentico tradimento di Cristo che è quello degli Apostoli. Da qui inizia un'opera di evangelizzazione contraria al messaggio dei Padri apostolici della Chiesa, che trasformerà Giuda nell'eretico degli eretici, colui che si oppone a Roma. Vassalli attraverso il dramma di un Giuda immemore prigioniero del tempo - evidente il richiamo alle Tres versiones de Judas di Borges - tradito nei suoi ideali, la Torah da parte di Cristo e il kerygma da parte delle Chiesa, rappresenta l'espressione di un disagio talvolta provato dall'uomo posto di fronte al Vangelo e a ciò che la Chiesa è stata ed è. La transattualizzazione del dramma di Giuda, come la modernizzazione della vicenda di Gesù di Saviane, ha appunto la funzione di attualizzare il dialogo con il messaggio di Cristo, rendendolo presente, resuscitandolo.

$R i$-scrivere il Vangelo è per questi autori quindi un modo per rinnovare, per adeguarlo alle istanze di una società emancipata dai dogmi, dando vita a un "cristianesimo possibile", in risposta all'eclissarsi del sacro e al suo ritorno in nuove forme. Il rinnovato "senso religioso" è innanzi tutto un'esperienza individuale di rapporto con la Scrittura e con Gesù, esperienza che dà vita a un nuovo interesse ermeneutico nei confronti dei Vangeli, a un discorso sull'uomo e sul rapporto che intrattiene con l'altro, e a un rifiuto dell'istituzione secolare della Chiesa. All'interno di questo "senso religioso" tutto post-moderno si colloca la figura di Giuda, come se nella figura del reietto gli autori proiettassero l'immagine reale dell'uomo contemporaneo nei suoi limiti e nelle sue imperfezioni. Ri-scrivere il Vangelo dal punto di vista di Giuda significa, quindi, tracciare i confini tra un messaggio come quello cristiano, per certi versi paradossalmente inattingibile per la ragione, e l'uomo, un essere finito, fragile, dominato dalla morte. 


\section{Bibliografia}

Berto G., La Gloria [1978], Milano, Mondadori, 200 .

—, La Passione secondo noi stessi, Milano, Rizzoli, 1972.

—, L'uomo e la sua morte [1962], Brescia, Morcelliana, 1964.

Boitani P., Ri-Scritture, Bologna, Il Mulino, 1997.

Bori P. C., L'interpretazione infinita, Bologna, Il Mulino, 1987.

Brelich M., L'opera del tradimento [1975], Milano, Adelphi, 1992.

Dотоцо C., Un cristianesimo possibile. Tra post-modernità e ricerca religiosa, Brescia, Queriniana, 2007.

Pazzi R., Il Vangelo di Giuda [1988], Milano, Baldini e Castoldi, I999.

Pomilio M., Il Quinto Evangelio [1975], Milano, Mondadori, I982.

—, Scritti cristiani, Milano, Rusconi, 1976.

Saviane G., Getsèmani, Milano, Mondadori, 1980.

Vassalli S., La notte del lupo, Milano, Baldini e Castoldi, 1989. 